\title{
Calculation of the filtration at the porous medium outlet
}

\author{
Yuri Osipov ${ }^{1, *}$, Galina Safina $^{1}$, and Nikita Vetoshkin $^{1}$ \\ ${ }^{1}$ Moscow State University of Civil Engineering, Yaroslavskoe shosse, 26, Moscow, 129337, Russia
}

\begin{abstract}
The study of fluid filtration with solid impurities in a porous medium is necessary for the construction of tunnels, hydraulic structures and underground storage of radioactive waste. The model of deep bed filtration of a monodisperse suspension in a homogeneous porous medium with variable porosity and permeability is considered. An asymptotic solution is constructed at the porous medium outlet. Calculations show the proximity of the high order asymptotics to the numerical solution.
\end{abstract}

\section{Introduction}

The filtration of a fluid with solid impurities in a porous medium is an important problem of underground hydromechanics. It is actual in the construction of tunnels, hydraulic structures, underground storage of radioactive waste [1-3]. Deep bed filtration of suspension in a porous medium determines the transport and deposition of solid particles on the porous medium frame. The deposit formation significantly changes the porosity, permeability and strength of the porous medium [4].

The transfer of particles in a porous medium is accompanied by the deposit formation. There are many different mechanisms for the particles retention: van der Waals forces, electrostatic interaction, adsorption, hydrodynamic forces, and the like. If pore size and suspended particles distributions overlap, the main mechanism for particles capture is sizeexclusion: solid particles pass freely through large pores, exceeding the diameter of the particles, and get stuck at the inlet of small pores with a cross-section smaller than the particle sizes [5]. A physical model of single pores clogging and particles capture is considered: one particle can block one small pore; one small pore can retain one solid particle. Assume that the retained particle cannot be knocked out of the pore by other particles or a fluid flow.

The mathematical model of suspension filtration includes the equation of the mass balance of suspended and retained particles and the kinetic equation of deposit growth [6]. The main characteristics of the porous medium - porosity and permeability are changed during the filtration process. The suspended particles move with a variable rate, depending on the size of the deposit. In the kinetic equation, the coefficient of proportionality between the rate of the deposit growth and the suspended particles concentration is called the filtration coefficient. The filtration coefficient is a decreasing function of the deposit concentration. If the filtration coefficient reaches 0 , then it is called blocking. The root of

*Corresponding author: yuri-osipov@mail.ru 
the filtration coefficient corresponds to the maximum value of the deposit concentration when all small pores are blocked by particles [7].

The asymptotic solution of the filtration problem at the porous medium outlet is constructed below. Asymptotic models of different orders are compared with a numerical solution. The analysis of the experimental data is carried out; the time intervals of the data applicability are obtained.

In Section 2 a mathematical model of deep bed filtration is considered. Section 3 is devoted to the construction of an asymptotic solution near the concentrations front of suspended and retained particles. An analysis of the experimental data is given in Section 4. The results of the numerical calculation are presented in Section 5. The conclusion in Section 6 finalizes the paper.

\section{Mathematical model}

The mathematical model of the one-dimensional filtration problem for a monodisperse suspension in a porous medium with variable porosity and permeability is determined by a quasilinear hyperbolic system of equations

$$
\begin{gathered}
\frac{\partial(g(S) C+S)}{\partial t}+\frac{\partial(f(S) C)}{\partial x}=0, \\
\frac{\partial S}{\partial t}=\Lambda(S) C .
\end{gathered}
$$

Here $C(x, t), S(x, t)$ are volumetric concentrations of suspended and retained particles, $\Lambda(S)$ is the filtration coefficient, $g(S), f(S)$ are variable porosity and permeability. Functions $g(S), f(S), \Lambda(S)$ are smooth and positive for $S \geq 0$.

The system of equations (1), (2) is considered in the domain

$$
\Omega=\{0<x<1, t>0\} .
$$

The boundary conditions for the system (1), (2) are set at the inlet of the porous medium $x=0$ and at the initial moment $t=0$ :

$$
\begin{aligned}
& \left.C(x, t)\right|_{x=0}=1 ; \\
& \left.C(x, t)\right|_{t=0}=0 ; \\
& \left.S(x, t)\right|_{t=0}=0 .
\end{aligned}
$$

A suspension of constant concentration is injected at the porous medium inlet (condition 3 ); at the initial moment the porous medium does not contain any particles (conditions 4,5 ). In laboratory experiments, the suspended particles concentration $C$ at the porous medium outlet is measured. To compare the theoretical calculations with experiment, the asymptotics of $C(x, t)$ at the outlet $x=1$ is most important.

The suspended particles are transported by the flow of the carrier fluid with a velocity $v=f(S) / g(S)$, and gradually the porous medium is filled by particles. The concentrations front of suspended and retained particles is the mobile two-phase boundary $\Gamma$ moving with constant velocity $v=f(0) / g(0)$. 
In the plane $x, t$ the concentrations front is a segment of straight line

$$
\{(x, t): t=\alpha x, 0 \leq x \leq 1\} ; \alpha=\frac{g(0)}{f(0)},
$$

which divides the domain $\Omega$ into two subdomains

$$
\Omega_{S}=\{0<x<1, t>x\} ; \Omega_{0}=\{0<x<1,0<t<x\}
$$

In the domain $\Omega_{0}$ the solution is zero: $C=0 ; S=0$; in the domain $\Omega_{S}$ the solution is positive: $C>0 ; S>0$. The solution $C(x, t)$ is discontinuous at the concentrations front $\Gamma$, the solution $S(x, t)$ is continuous in $\Omega$.

The exact solution of the problem (1)-(5) at the concentrations front has the form [8]

$$
\left.C(x, t)\right|_{\Gamma}=\frac{1}{\left(\alpha f_{1}-g_{1}\right)+\left(1+g_{1}-\alpha f_{1}\right) e^{\frac{\lambda_{0}}{f_{0}} x}} ;\left.S(x, t)\right|_{\Gamma}=0 .
$$

\section{Asymptotic solution near the concentrations front}

Assume that in a neighborhood of a point $S=0$ the functions $g(S), f(S), \Lambda(S)$ can be expanded in the form

$$
\begin{gathered}
g(S)=g_{0}+g_{1} S+g_{2} S^{2}+\ldots, g_{0}>0 ; \\
f(S)=f_{0}+f_{1} S+f_{2} S^{2}+\ldots, f_{0}>0 ; \\
\Lambda(S)=\lambda_{0}+\lambda_{1} S+\lambda_{2} S^{2}+\ldots, \lambda_{0}>0 ;
\end{gathered}
$$

In the domain $\Omega_{S}$ near the concentrations front the asymptotic solution is constructed in the form of series in powers of a small parameter $t-\alpha x$ :

$$
\begin{gathered}
C(x, t)=c_{0}(x)+(t-\alpha x) c_{1}(x)+(t-\alpha x)^{2} c_{2}(x)+\ldots ; \\
S(x, t)=(t-\alpha x) s_{0}(x)+(t-\alpha x)^{2} s_{1}(x)+(t-\alpha x)^{3} s_{2}(x)+\ldots ;
\end{gathered}
$$

Here the principal asymptotic term $c_{0}(x)$ of the suspended particles concentration is given by formula (6).

Substitution of the expansions (10), (11) into equation (1) and equating the expressions for the same powers $(t-\alpha x)$ yields the system of differential equations

$$
\begin{gathered}
(t-\alpha x)^{0}: g_{1} s_{0}(x) c_{0}(x)+s_{0}(x)+c_{0}^{\prime}(x) f_{0}-\alpha f_{1} s_{0}(x) c_{0}(x)=0 \\
2\left[g_{1} s_{0}(x) c_{1}(x)+g_{1} s_{1}(x) c_{0}(x)+g_{2} s_{0}^{2}(x) c_{0}(x)+s_{1}(x)\right]+ \\
(t-\alpha x)^{1}:+\left[f_{1} s_{0}^{\prime}(x) c_{0}(x)+f_{1} s_{0}(x) c_{0}^{\prime}(x)+f_{0} c_{1}^{\prime}(x)\right] \\
-2 \alpha\left[f_{1} s_{1}(x) c_{0}(x)+f_{2} s_{0}^{2}(x) c_{0}(x)+f_{1} s_{0}(x) c_{1}(x)\right]=0
\end{gathered}
$$




$$
\begin{aligned}
& 3\left(g_{1} s_{0}(x) c_{2}(x)+g_{1} s_{1}(x) c_{1}(x)+g_{2} s_{0}^{2}(x) c_{1}(x)+\right. \\
& \left.+g_{1} s_{2}(x) c_{0}(x)+2 g_{2} s_{0}(x) s_{1}(x) c_{0}(x)+g_{3} s_{0}{ }^{3}(x) c_{0}(x)+s_{2}(x)\right)+ \\
(t-\alpha x)^{2}: & +\left(f_{1} s_{1}^{\prime}(x) c_{0}(x)+f_{1} s_{1}(x) c_{0}^{\prime}(x)+2 f_{2} s_{0}(x) s_{0}^{\prime}(x) c_{0}(x)+f_{2} s_{0}{ }^{2}(x) c_{0}^{\prime}(x)+\right. \\
& \left.+f_{1} s_{0}^{\prime}(x) c_{1}(x)+f_{1} s_{0}(x) c_{1}^{\prime}(x)+f_{0} c_{2}^{\prime}(x)\right)- \\
& -3 \alpha\left(f_{1} s_{2}(x) c_{0}(x)+2 f_{2} s_{0}(x) s_{1}(x) c_{0}(x)+f_{3} s_{0}{ }^{3}(x) c_{0}(x)+f_{1} s_{1}(x) c_{1}(x)+\right. \\
& \left.+f_{2} s_{0}{ }^{2}(x) c_{1}(x)+f_{1} s_{0}(x) c_{2}(x)\right)=0
\end{aligned}
$$

Substitution of (10), (11) into equation (2) yields the algebraic relations

$$
\begin{gathered}
(t-\alpha x)^{0}: s_{0}=\lambda_{0} c_{0} \\
(t-\alpha x)^{1}: 2 s_{1}=\lambda_{0} c_{1}+\lambda_{1} s_{0} c_{0} \\
(t-\alpha x)^{2}: 3 s_{2}=\lambda_{0} c_{2}+\lambda_{1}\left(s_{0} c_{1}+s_{1} c_{0}\right)+\lambda_{2} s_{0}^{2} c_{0}
\end{gathered}
$$

The uniqueness of the solutions $c_{i}(x)$ of the differential equations (12)-(14) is determined by the boundary conditions, which follow from (3):

$$
\left.c_{i}(x)\right|_{x=0}=0 .
$$

The main terms of the asymptotics are determined from equations (12), (13), (15), (16). Substitution of the asymptotic expansions into (10), (11) gives

$$
\begin{aligned}
& C(x, t)=c_{0}(x)+\left(\lambda_{0} \alpha_{2}\left(1-e^{2 k x}\right)+\left(1+\alpha_{1}\right)\left(\lambda_{1}-2 k f_{1}\right) e^{k x}\left(1-e^{k x}\right)\right) c_{0}^{3}(x)(t-\alpha x) \\
& S(x, t)=\lambda_{0} c_{0}(x)(t-\alpha x)+ \\
& +\frac{\lambda_{0}}{2}\left(\alpha_{1} e^{k x}\left(2\left(\lambda_{1}-k f_{1}\right)-\left(\lambda_{1}-2 k f_{1}\right) e^{k x}\right)+\lambda_{0} \alpha_{2}\left(1-e^{2 k x}\right)-\lambda_{1} \alpha_{1}\right) c_{0}^{3}(x)(t-\alpha x)^{2}
\end{aligned}
$$

Here

$$
\alpha_{1}=g_{1}-\alpha f_{1}, \alpha_{2}=g_{2}-\alpha f_{2}, k=\lambda_{0} / f_{0}
$$

are constants.

The next terms of the asymptotics cannot be written out in general form because of the awkwardness of the expressions. However, they can be calculated for specific coefficients of equations (1), (2) for $x=1$. The asymptotic solution at the porous medium outlet in compact form is obtained in Section 5.

\section{Analysis of the laboratory experiments}

The coefficients of the mass balance and the kinetic growth equations (1), (2) were obtained by Z. You on the basis of laboratory experiments. Deep bed filtration of monodisperse suspensions containing solid particles of three sizes was studied [8]. The results are collected in Table 1. 
Table 1. The equations coefficients measured in laboratory experiments

\begin{tabular}{|c|c|c|}
\hline Type & $\begin{array}{c}\text { The particle } \\
\text { radius, } \mu \mathrm{m}\end{array}$ & \multicolumn{1}{|c|}{ The equations coefficients } \\
\hline \multirow{3}{*}{1} & \multirow{3}{*}{$r_{1}=1.5675$} & $g_{1}(S)=0.9987+9.1 \cdot 10^{-13} S-3.73 \cdot 10^{-8} S^{2}+6.1 \cdot 10^{-5} S^{3}$ \\
\cline { 3 - 3 } & & $f_{1}(S)=0.9999+1.8 \cdot 10^{-5} S-2.05 \cdot 10^{-7} S^{2}+2.848 \cdot 10^{-4} S^{3}$ \\
\cline { 3 - 3 } & $\Lambda_{1}(S)=0.11-0.01351 \cdot S+4.49 \cdot 10^{-5} S^{2}+1.163 \cdot 10^{-3} S^{3}$ \\
\hline \multirow{3}{*}{2} & \multirow{3}{*}{$r_{2}=2.179$} & $g_{2}(S)=0.9743-8.88 \cdot 10^{-14} S+1.27 \cdot 10^{-11} S^{2}-1.24 \cdot 10^{-9} S^{3}$ \\
\cline { 3 - 3 } & & $f_{2}(S)=0.9947+6.27 \cdot 10^{-5} S-2.9 \cdot 10^{-8} S^{2}+6.21 \cdot 10^{-10} S^{3}$ \\
\cline { 3 - 3 } & & $\Lambda_{2}(S)=0.51-5.956 \cdot 10^{-3} S+2.29 \cdot 10^{-6} S^{2}+1.35 \cdot 10^{-8} S^{3}$ \\
\hline \multirow{3}{*}{3} & \multirow{3}{*}{$r_{3}=3.168$} & $g_{3}(S)=0.7635+2.44 \cdot 10^{-15} S+3.2 \cdot 10^{-14} S^{2}+3.6 \cdot 10^{-13} S^{3}$ \\
\cline { 3 - 3 } & & $f_{3}(S)=0.9075+2.315 \cdot 10^{-4} S+2.27 \cdot 10^{-8} S^{2}-3.42 \cdot 10^{-8} S^{3}$ \\
\cline { 3 - 3 } & & $\Lambda_{3}(S)=1.551-3.467 \cdot 10^{-3} S-1.16 \cdot 10^{-6} S^{2}-1.16 \cdot 10^{-7} S^{3}$ \\
\hline
\end{tabular}

Analysis of the filtration coefficients shows that the function $\Lambda_{1}(S)$ decreases to the minimum point $S_{0}=1.95$, and then increases. The exact solution at the inlet $x=0$ [9]

$$
\int_{0}^{S\left(0, t_{0}\right)} \frac{d S}{\Lambda(S)}=t_{0}
$$

allows to determine the moment of minimum concentration $S_{0}: t_{0}=19.6$. Taking into account the time $\Delta t=1$ of motion of the concentrations front from the inlet of the porous medium to the outlet, we obtain the time interval for the applicability of the experimental data for particles of type 1 for $x=1: 1 \leq t \leq 20.6$.

The filtration coefficients $\Lambda_{2}(S), \Lambda_{3}(S)$ decrease to zero, therefore, at the outlet the experimental data for particles of types 2 and 3 are applicable for $1 \leq t<\infty$.

\section{Numerical modelling}

The numerical calculation of the solution is performed by the method of finite differences using an explicit difference scheme with steps matched on the concentrations front $t=\alpha x$ : $h_{x}=0.001 ; h_{t}=\alpha h_{x}$ similarly to [10]. Calculation of the asymptotics for particles of types 1,2 and 3 at the porous medium outlet up to the third order was performed using the Wolfram Mathematics software package. The asymptotic formulas are given below.

$$
\begin{aligned}
& C_{1}(1, t)=0.8958+0.001261(t-0.9988)-6.5051 \cdot 10^{-6}(t-0.9988)^{2} \\
& S_{1}(1, t)=0.09854(t-0.9988)-0.0005269(t-0.9988)^{2}+1.4578 \cdot 10^{-6}(t-0.9988)^{3} \\
& C_{2}(1, t)=0.5988+0.001446(t-0.9795)-1.1264 \cdot 10^{-6}(t-0.9795)^{2} \\
& S_{2}(1, t)=0.3054(t-0.9795)-0.0001759(t-0.9795)^{2}-8.1664 \cdot 10^{-7}(t-0.9795)^{3} \\
& C_{3}(1, t)=0.1811+0.0006315(t-0.8413)+8.8487 \cdot 10^{-7}(t-0.8413)^{2} \\
& S_{3}(1, t)=0.2808(t-0.8413)+0.0004016(t-0.8413)^{2}+1.6298 \cdot 10^{-7}(t-0.8413)^{3}
\end{aligned}
$$


In Figures 1-6 the graphs of the numerical solution (solid line) and asymptotic expansions of orders 1 (dashed line), 2 (dotted line), and 3 (dot-dash line) for the suspended and retained particles of 1,2 and 3 types at the outlet are shown. The time interval for particles of types 2 and 3 is chosen so that the asymptotics of the third order provides the best approximation of the solution.

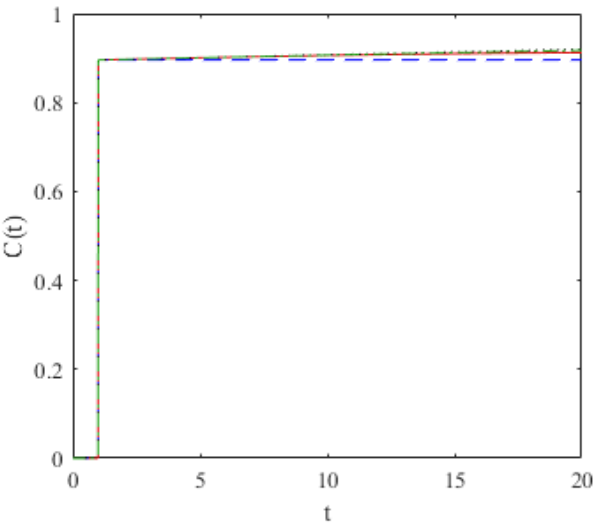

Fig. 1. Type 1 a) general view of $\left.C_{1}(x, t)\right|_{x=1}$

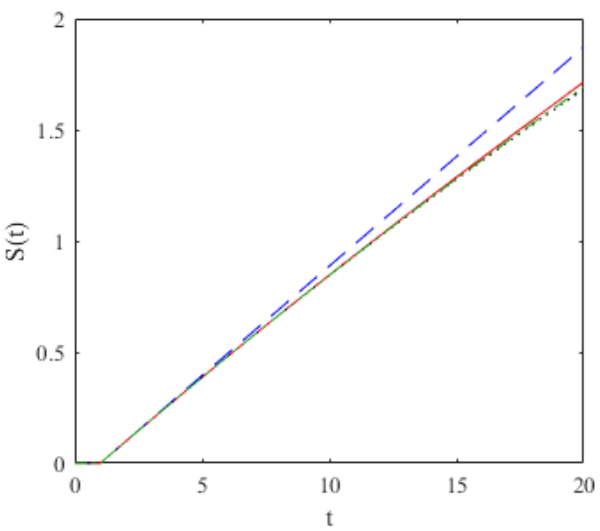

Fig. 2. Type 1 a) general view of $\left.S_{1}(x, t)\right|_{x=1}$

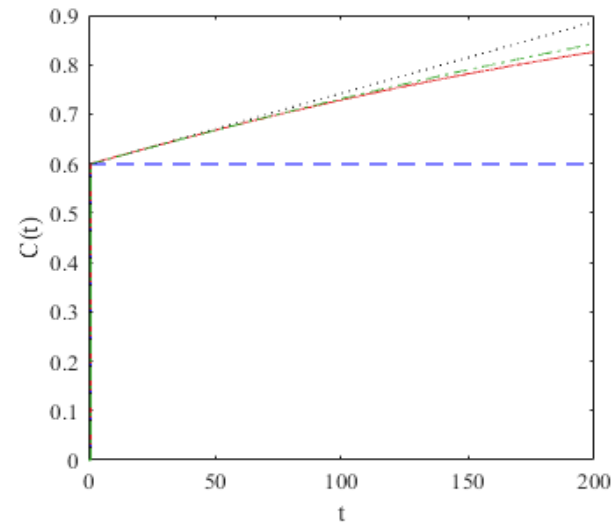

Fig. 3. Type 2 a) general view of $\left.C_{2}(x, t)\right|_{x=1}$

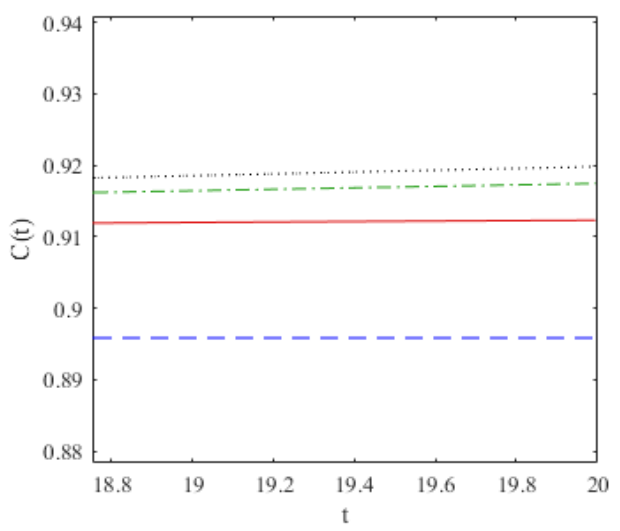

b) enlarged view of $\left.C_{1}(x, t)\right|_{x=1}$

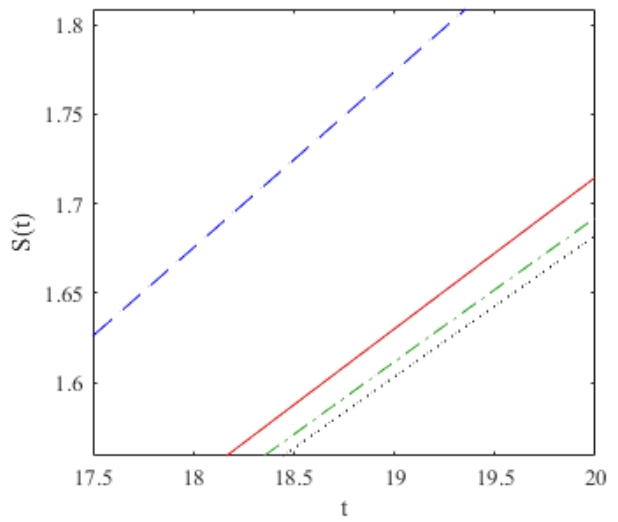

b) enlarged view of $\left.S_{1}(x, t)\right|_{x=1}$

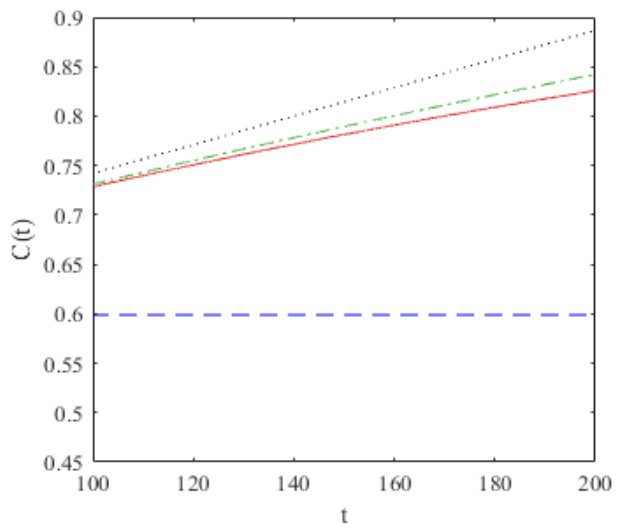

b) enlarged view of $\left.C_{2}(x, t)\right|_{x=1}$ 


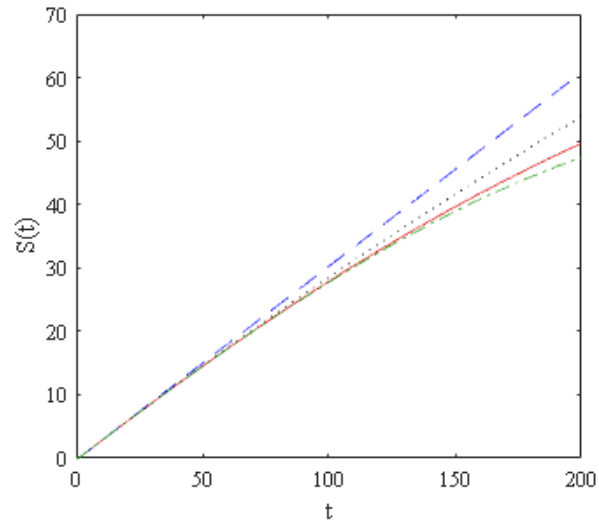

Fig. 4. Type 2 a) general view of $\left.S_{2}(x, t)\right|_{x=1}$

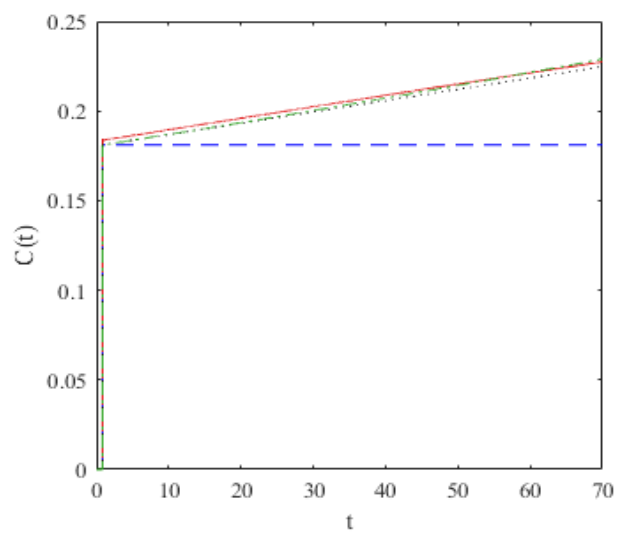

Fig. 5. Type 3 a) general view of $\left.C_{3}(x, t)\right|_{x=1}$

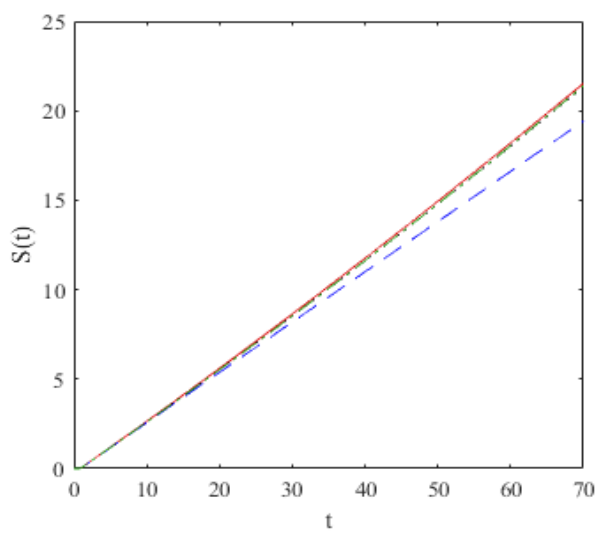

Fig. 6. Type 3 a) general view of $\left.S_{3}(x, t)\right|_{x=1}$

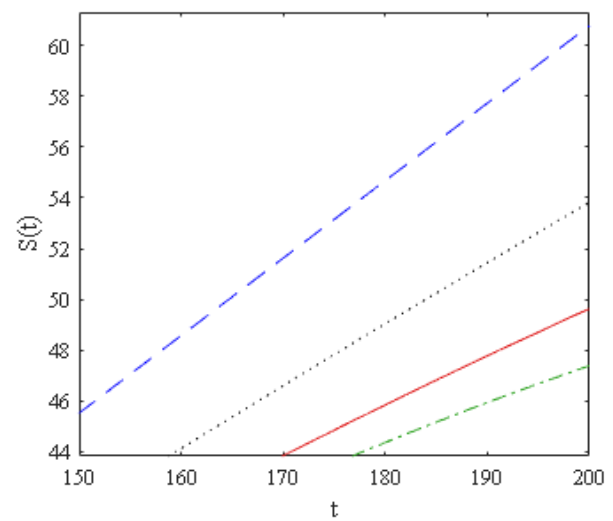

b) enlarged view of $\left.S_{2}(x, t)\right|_{x=1}$

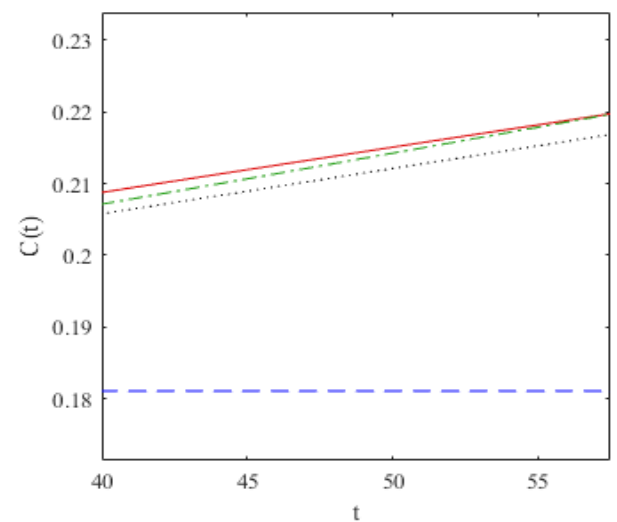

b) enlarged view of $\left.C_{3}(x, t)\right|_{x=1}$

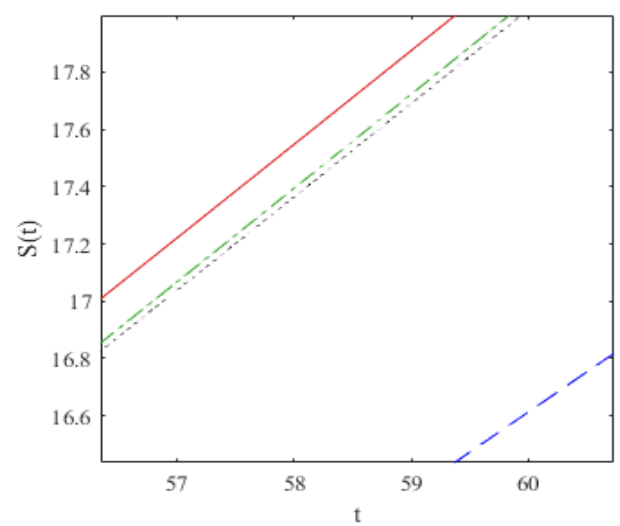

b) enlarged view of $\left.S_{3}(x, t)\right|_{x=1}$

The Figures 1-6 show that the asymptotics rapidly approach the solution with increasing order. The difference between the numerical solution and the third-order asymptotics is less than $2 \%$ on the whole time interval. 


\section{Conclusion}

The analysis of the experimental data shows that the filtration model (1)-(5) is applicable for particles of type 1 in a limited time interval $0 \leq t \leq 20.6$; for particles of types 2 and 3 , the time interval is unlimited.

For the filtration problem in a porous medium with variable porosity and permeability, the asymptotic terms of 2 and 3 orders cannot be written out in general form because of cumbersome expressions. However, for specific values of the parameters given in Table 1, the asymptotic formulas at the porous medium outlet have a compact form and can be used to approximate the solution.

For the filtration problem of a monodisperse suspension with solid particles of 3 different sizes, asymptotic solutions of various orders are constructed at the porous medium outlet. It is shown that as the number of terms increases, the asymptotics becomes closer to the solution. The best approximation is given by an asymptotic solution of order 3 .

\section{References}

1. Li S, Liu R, Zhang Q and Zhang, Protection against water or mud inrush in tunnels by grouting: a review, J. of Rock Mechanics and Geotechnical Engineering, 8, pp 753766, (2016)

2. Tsuji M, Kobayashi S, Mikake S, Sato T and Matsui H, Post-Grouting Experiences for Reducing Groundwater Inflow at $500 \mathrm{~m}$ Depth of the Mizunami Underground Research Laboratory, Japan. Procedia Engineering, 191, 543-550, (2017)

3. Yoon J and Mohtar C S El, Groutability of Granular, Soils Using Bentonite Grout Based on Filtration Model, Transport in Porous Media, 102(3), pp 365-385, (2014)

4. Civan F. Reservoir Formation Damage: Fundamentals, Modeling, Assessment and Mitigation. 2nd ed., (Gulf Professional Pub., Amsterdam, 2007)

5. Santos A, Bedrikovetsky P and Fontoura S, Analytical micro model for size exclusion: Pore blocking and permeability reduction, J. of Membrane Science, 308, pp 115-127, (2008)

6. You Z, Bedrikovetsky P and Kuzmina L, Exact solution for long-term size exclusion suspension-colloidal transport in porous media, Abstract and Applied Analysis, article ID 680693, (2013)

7. Kuzmina L I and Osipov Yu V, Asymptotic solution for deep bed filtration with small deposit, Procedia Engineering 111 491-494, (2015)

8. You Z, Osipov Y, Bedrikovetsky P and Kuzmina L, Asymptotic model for deep bed filtration Chemical Engineering Journal, 258 pp 374-385, (2014)

9. Vyazmina E A, Bedrikovetskii P G and Polyanin A D, New classes of exact solutions to nonlinear sets of equations in the theory of filtration and convective mass transfer, Theoretical Foundations of Chemical Engineering, 41(5) pp 556-564, (2007)

10. Galaguz Y P and Safina G L, Modeling of fine migration in a porous medium, MATEC Web of Conferences, 86, 03003, (2016) 